\title{
Clinical utility of pembrolizumab in the management of advanced solid tumors: an evidence-based review on the emerging new data
}

This article was published in the following Dove Press journal: Cancer Management and Research

\section{Pauline du Rusquec Ombline de Calbiac Marie Robert Mario Campone Jean Sebastien Frenel}

Medical Oncology Department, Institut de Cancérologie de l'Ouest, SaintHerblain 44800, France
Correspondence: Jean Sebastien Frenel Medical Oncology Department, Institut de Cancérologie de l'Ouest, II Boulevard Jacques Monod, Saint-Herblain 44800

France

Tel +33240679900

Fax +3 3240679776

Email Jean-Sebastien.Frenel@ico.unicancerffr

\begin{abstract}
Pembrolizumab is a full-length human immunoglobulin G4 (IgG4) monoclonal antibody directed against the immune checkpoint PD-1 to remove its binding with PD-L1 and thus to restore an anti-tumor immune response of T cells. Pembrolizumab is one of the most advanced immune checkpoint inhibitors for cancer care. Apart from rare and serious adverse effects, its favorable tolerance profile enables to treat fragile patients who have often no other choice than best supportive care. The effective retained dose of pembrolizumab is a venous administration of $200 \mathrm{mg}$ every 3 weeks until disease progression, intolerance or up to 24 months. Pembrolizumab has already proven its efficacy and thus obtained marketing authorization in so-called hot or hypermutated tumors or tumors expressing PD-L1 such as melanomas, non-small cell lung cancers, urothelial carcinomas, cervical cancer, etc. Pembrolizumab is also authorized in the United States in the treatment of mismatch repairdeficient tumors or with microsatellite instability. The current challenge is to expand its use in tumor types that are supposed to be less immunogenic, for example, by attempting to warm up the tumor microenvironment, or by combining pembrolizumab with other molecules. An acceptable toxicity profile of such combinations remains to explore. We review here the current indications of this drug, the main prognostic and predictive factors of its efficacy as well as the potential forthcoming indications.
\end{abstract}

Keywords: pembrolizumab, immune checkpoint inhibitor, anti PD-1 antibody

\section{Introduction}

Immunotherapy is a major breakthrough of cancer therapy in recent years, as shown by the awarding of the 2018 Nobel Prize in Medicine to immunologist James Allison. To restore the patient's own anti-tumor immunity is now a successful strategy in opposition to conventional cytotoxic or targeted therapy. Pembrolizumab is a major weapon in the immunotherapy pipeline, and we review here the latest data on its development.

\section{Pembrolizumab}

Pembrolizumab (MK3475, Keytruda ${ }^{\circledR}$, Merck \& Co., ${ }^{1}$ Whitehouse Station, New Jersey, USA) is a full-length human immunoglobulin G4 (IgG4) monoclonal antibody directed against the immune checkpoint Programmed cell Death 1.

\section{a. Mechanism of action}

Programmed cell Death 1 (PD-1) is expressed on activated T cells acting as a checkpoint of the effector stage of the immune response (Figure 1). ${ }^{2}$ Its ligands, Programmed cell 


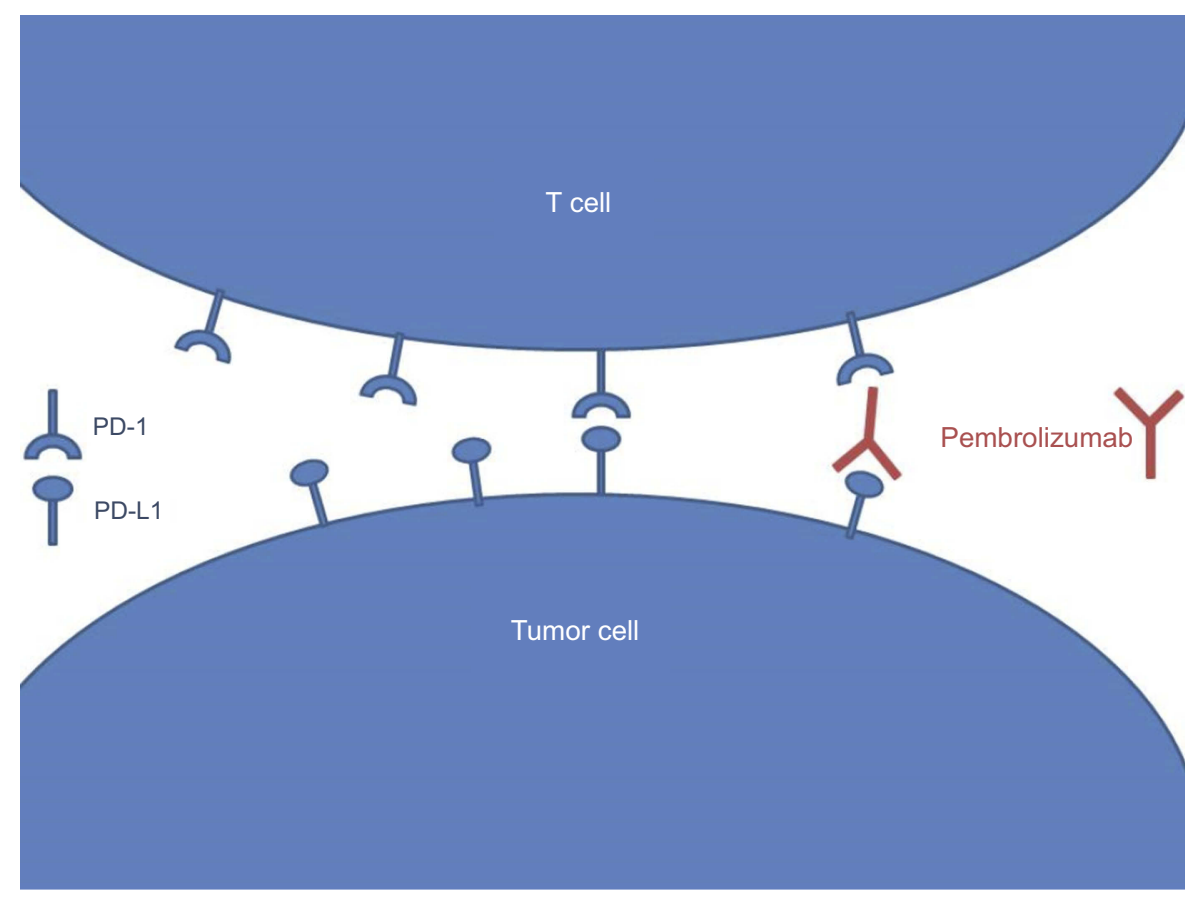

Figure I Modeling the mode of action of pembrolizumab.

Death Ligand 1 (PD-L1) and 2 (PD-L2), are expressed on tumor cells, macrophages, and dendritic cells. ${ }^{3}$ The binding of PD1 with PD-L1 triggers the tolerance of tumor cells by the immune system, promoting tumor growth. By impairing the PD-1/PD-L1 binding, Pembrolizumab leads to a physiological shift to immune reactivity and anti-tumor effect.

\section{b. Pharmacokinetics}

Pembrolizumab is supplied as powder for solution for infusion as $100 \mathrm{mg} / 4 \mathrm{~mL}$ vials for intravenous (IV) injection. ${ }^{4}$ Pembrolizumab is administered intravenously at $200 \mathrm{mg}$ every 3 weeks, immediately and completely bioavailable. The time to reach steady state is of 18 weeks. Age, gender, race, and tumor burden have no clinically meaningful effect on clearance like mild or moderate renal impairment or mild hepatic impairment. ${ }^{5,6}$

\section{Studies and clinical development}

Developmental studies of pembrolizumab are usually preceded by the acronym KEYNOTE. Dozens of studies are in progress, in monotherapy or combination in almost all types of cancer. First in list, KEYNOTE-001 demonstrated the activity of pembrolizumab in non-small cell lung cancer (NSCLC), ${ }^{7}$ and highlighted the importance of PD-L1 expression as predictive biomarker. A pooled analysis of 14 trials has shown an overall response rate
(ORR) of $26 \%$ with this drug (95\% Confidence Interest (CI) 21 to 31$)^{8}$

\section{d. Tolerance of pembrolizumab}

The safety profile is satisfactory and superimposable with other checkpoint inhibitors. Overall, the incidence of any grade treatment adverse events (AE) in 3922 patients was $74.3 \%$ (95\% CI 0.671 to 0.805$)$. ${ }^{9}$ A meta-analysis of 11 studies found $9 \%$ (95\% CI $6 \%$ to $14 \%$ ) of grade $3 / 4 \mathrm{AE},{ }^{8}$ significantly lower than conventional treatments. Overall, $5 \%$ of the patients went out of clinical trial for unacceptable toxicity. The most common $\mathrm{AE}$ are fatigue, diarrhea, nausea, rash and pruritus, myalgia and arthralgia. Less frequent but more concerning $\mathrm{AE}$ are the so-called immune-related Adverse Events (irAEs) related to the therapeutic class of pembrolizumab and generally consistent across tumor types. ${ }^{10}$ Endocrine irAES are the most frequent, with $10-15 \%$ of hypothyroidism/hyperthyroidism and $1-3 \%$ of hypophysitis $(1-3 \%) .{ }^{11,12}$ Some rare but life-threatening $\mathrm{AE}$ have been reported with like encephalopathy, ${ }^{13}$ pneumonitis, ${ }^{14}$ nephritis, ${ }^{15}$ hepatitis, ${ }^{16}$ myocarditis, ${ }^{17}$ and colitis. ${ }^{18}$ Their management includes the suspension of the drug, substitutive opotherapy and immunosuppression by high doses of corticosteroids or powerful immunosuppressant like tumor necrosis factor antagonists or mycophenolate mofetil. 


\section{Approved indications for pembrolizumab}

\section{a. Melanoma}

Table 1 summarize current approved indications for pembrolizumab. In 2015, pembrolizumab monotherapy was the first anti-PD1 antibody approved in Europe for treating advanced melanoma progressing on standard therapy based on data from KEYNOTE-001 and KEYNOTE002. ${ }^{19,20}$ The KEYNOTE-006 comparing pembrolizumab to ipilimumab in first or second line leads to an extension of this approval for previously untreated melanoma regardless of BRAF status. ${ }^{21}$ This trial showed an impressive overall survival (OS) benefit for pembrolizumab: 32.7 vs 15.9 months (HR 0.73 (95\%CI 0.61 to 0.89$)$ ). Among the 103 patients who continued 2 years of pembrolizumab, $86 \%$ were free of progression 20 months after discontinuation of the drug. Recently, the FDA has accepted a supplemental biologics license application (sBLA) for the use of pembrolizumab as an adjuvant treatment for patients with high-risk resected stage III melanoma, based on the KEYNOTE-054 trial. $^{36}$ Hazard ratio for recurrence or death was $0.57(98.4 \% \mathrm{CI}, 0.43$ to 0.74$)$, $P<0.001$.

\section{b. Lung cancer NSCLC}

Following KEYNOTE-010, ${ }^{22}$ pembrolizumab was approved in 2015 in second line for PD-L1-positive (Tumor proportion score (TPS) $\geq 1 \%$ ) NSCLC pretreated by chemotherapy or tyrosine kinase inhibitor (TKI) if epidermal growth factor receptor (EGFR) mutated or anaplastic lymphoma kinase (ALK)rearranged. Based on KEYNOTE-024 trial, ${ }^{23}$ indication was extended in 2016 to front line EGFR/ALK wild type PD-L1+ (TPS $\geq 50 \%)$ NSCLC. Of note, the KEYNOTE-042, ${ }^{37,24}$ in the same context but with a different PD-L1 expression (TPS score $\geq 1 \%$ ), did not show any benefit of pembrolizumab vs chemotherapy in that broader population. More recently, a combination with pemetrexed and platinum chemotherapy as first line has been approved based on an OS improvement in the KEYNOTE-189 trial. $^{25}$ OS benefit was seen across all PD-L1 categories of PD-L1 expression.

\section{Squamous cell NSCLC}

Pembrolizumab plus carboplatin/paclitaxel or nabpaclitaxel should become a new standard of care for the first-line treatment base on significant OS benefit in the KEYNOTE-407. ${ }^{26}$ Interestingly, the grade $3 \mathrm{AE}$ was lower in the combination arm (74.5\% vs $64.4 \%)$. Pembrolizumab benefit was independent from PD-L1 TPS expression, but the magnitude of the benefit was correlated with PD-L1 expression.

\section{c. Bladder cancer}

The KEYNOTE-045 leads to the FDA approval of pembrolizumab for urothelial carcinoma progressing after platinum comparing pembrolizumab with investigator's choice chemotherapy in second-line. ${ }^{27}$ Interestingly the PD-L1-positive ( $\geq 10 \%$ ) subgroup seems to have a worst prognosis than the global population: median OS, 8.0 months vs 5.2 months ( $\mathrm{HR}=0.57$ (95\% CI, 0.37 to 0.88$)$; $P=0.005)$. Pembrolizumab is also approved for cisplatinineligible patients based on the KEYNOTE-052 and then offers a real hope to a group of patient with a very poor prognosis. $^{28,38}$

\section{d. Cervical cancer}

In June 2018, Pembrolizumab has been granted approval by the FDA for the treatment of patients with advanced, PD-L1+ cervical cancer progressing after chemotherapy. The KEYNOTE-028 multi-cohort phase Ib trial included 24 heavily pretreated patients with $\mathrm{PD}-\mathrm{L} 1 \geq 1 \%$ cervical cancer to receive pembrolizumab $10 \mathrm{mg} / \mathrm{kg}$ Q2W for up to 24 months. ${ }^{39}$ ORR was $17 \%$ with a median DOR of 5.4 months. Then, the phase II basket KEYNOTE-158 study of 11 cancer types enrolled 98 patients with pretreated cervical cancer to receive pembrolizumab. ${ }^{30}$ Results showed an ORR of $13.3 \%$ with responses restricted to PD-L1+ tumors.

\section{e. Gastrointestinal cancers}

\section{Gastric cancer}

The KEYNOTE-059 phase II trial included advanced gastric cancer ( $\geq 2$ lines). ${ }^{31}$ ORR was $11.6 \%$. ORR was $15.5 \%$ and $6.4 \%$ for PD-L1-positive and negative patients, respectively. Given the benefit in RR and DOR, the FDA has granted marketing authorization for patients with PD-L1+ gastric cancer on the 3 rd or higher line. ${ }^{40}$ The phase III KEYNOTE-061 failed to confirm the superiority of pembrolizumab to paclitaxel chemotherapy as a second-line therapy with post-hoc interesting results in MSI high tumors. ${ }^{32}$ The ongoing phase III trial KEYNOTE-062 is testing the frontline combination of pembrolizumab + Cisplatinum/5FU in PD-L1+ and HER2 negative tumors. ${ }^{41}$ 


\begin{tabular}{|c|c|c|c|c|c|}
\hline 畜 & $\frac{9}{\frac{9}{2}}$ & & $\stackrel{n}{\grave{I}}$ & $\frac{n}{\bar{a}}$ & 음 \\
\hline 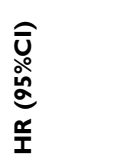 & 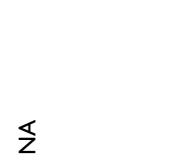 & $\mathbb{z}$ & 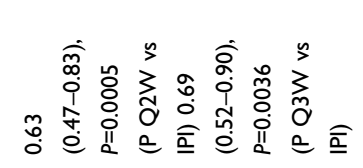 & \multicolumn{2}{|c|}{ 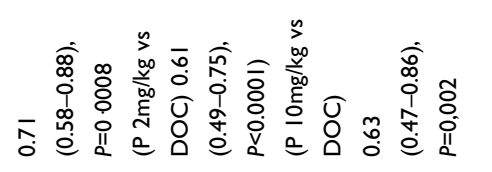 } \\
\hline 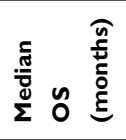 & 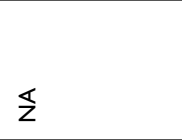 & $\mathbb{z}$ & $\frac{\alpha}{z}$ & 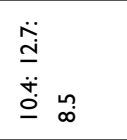 & 先 \\
\hline 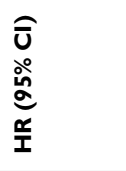 & 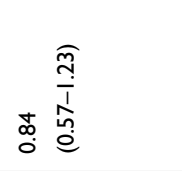 & \multicolumn{2}{|c|}{ 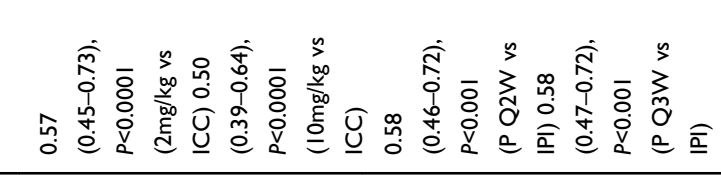 } & \multicolumn{2}{|c|}{ 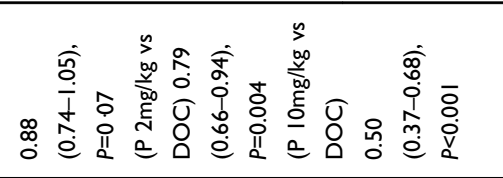 } \\
\hline 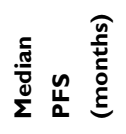 & \multicolumn{2}{|c|}{ 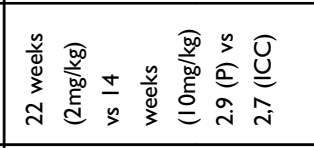 } & 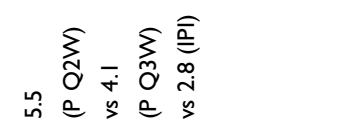 & $\begin{array}{l}\ddot{\sigma} \\
\dot{+} \\
\dot{m}\end{array}$ & 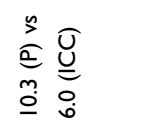 \\
\hline$\frac{x}{\tilde{c}}$ & 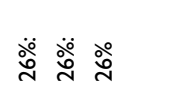 & 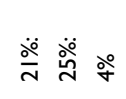 & 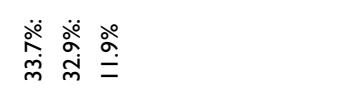 & $\stackrel{\circ}{\circ} \stackrel{\circ}{\circ} \stackrel{\circ}{\circ}$ & 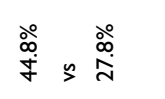 \\
\hline 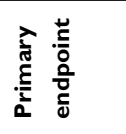 & 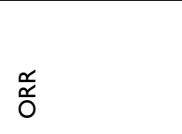 & 씀 & 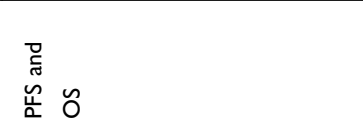 & 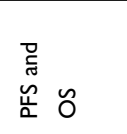 & 营 \\
\hline 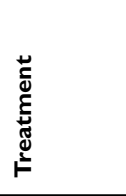 & 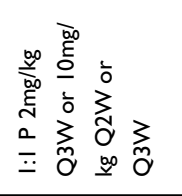 & 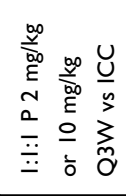 & 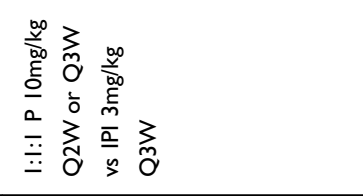 & 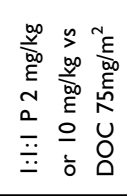 & 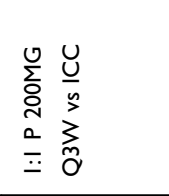 \\
\hline 岂 & $\pi$ & $\tilde{\lambda}$ & $\underline{I}$ & $\tilde{\Lambda}$ & $\underline{\underline{n}}$ \\
\hline$=$ & 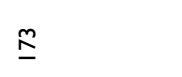 & 움 & $\underset{\infty}{ \pm}$ & 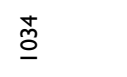 & 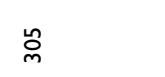 \\
\hline 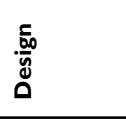 & 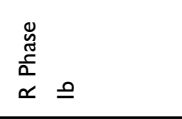 & 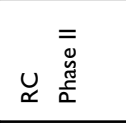 & 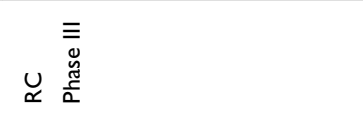 & 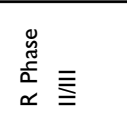 & 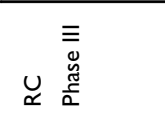 \\
\hline 空 & 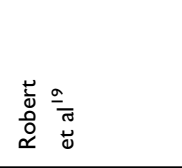 & 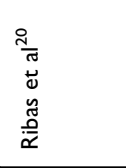 & 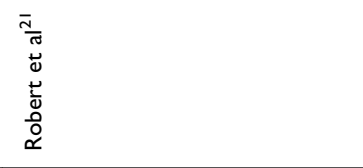 & 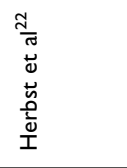 & 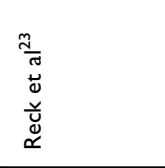 \\
\hline 总 & $\frac{\text { à }}{4}$ & $\stackrel{n}{a}$ & $\stackrel{n}{\bar{i}}$ & $\stackrel{\circ}{\circ}$ & $\stackrel{2}{2}$ \\
\hline 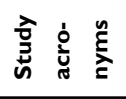 & $\overline{8}$ & ธั & ః̊ & 응 & ఫ̃ \\
\hline 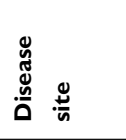 & 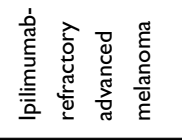 & & 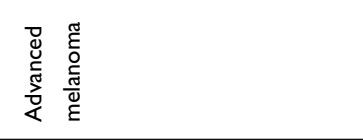 & 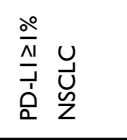 & 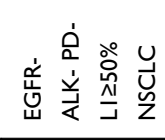 \\
\hline 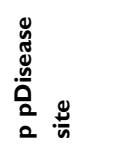 & 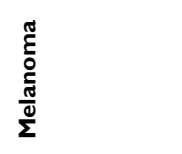 & & & $\stackrel{\infty}{\Xi}$ & \\
\hline
\end{tabular}




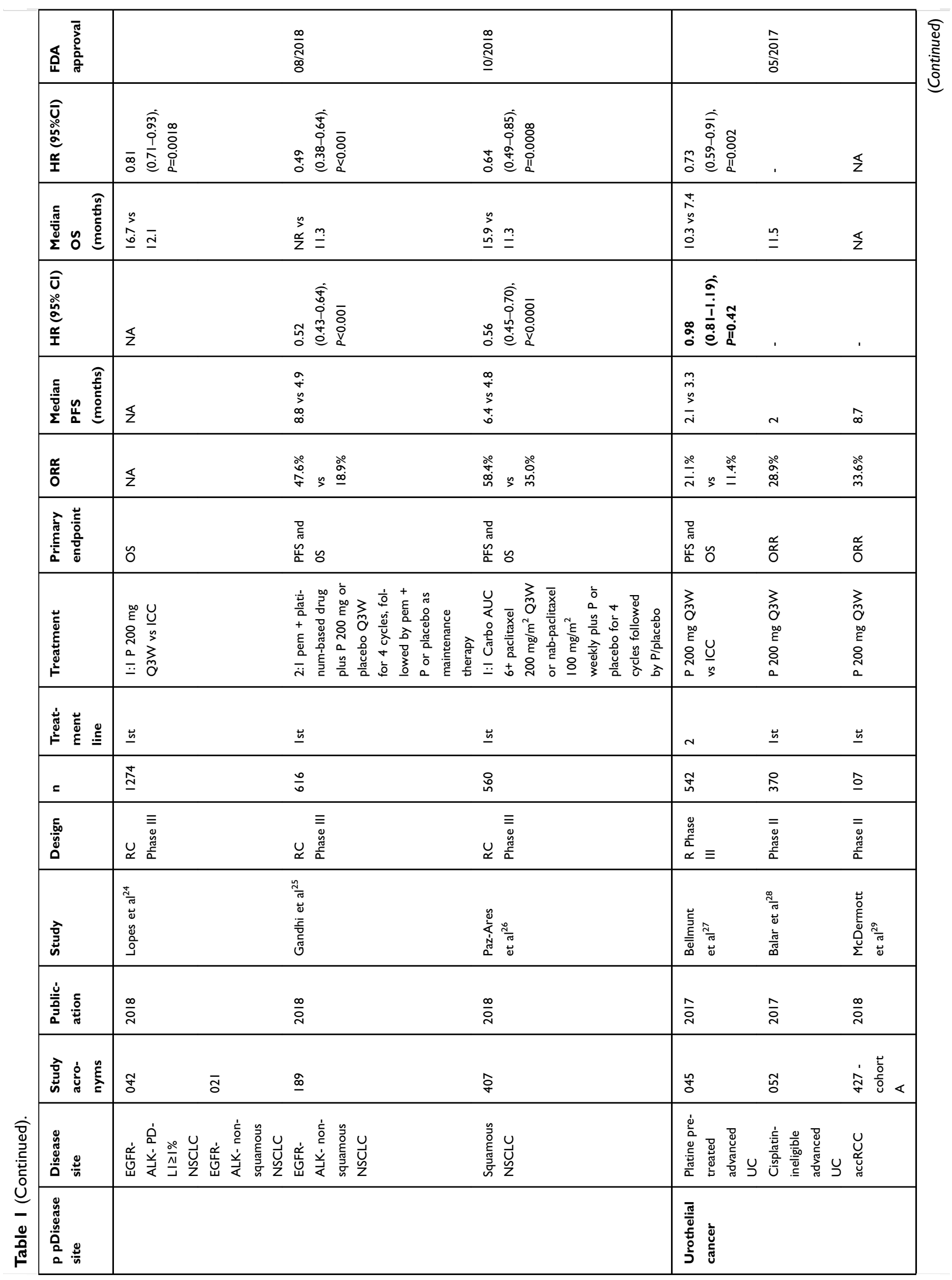




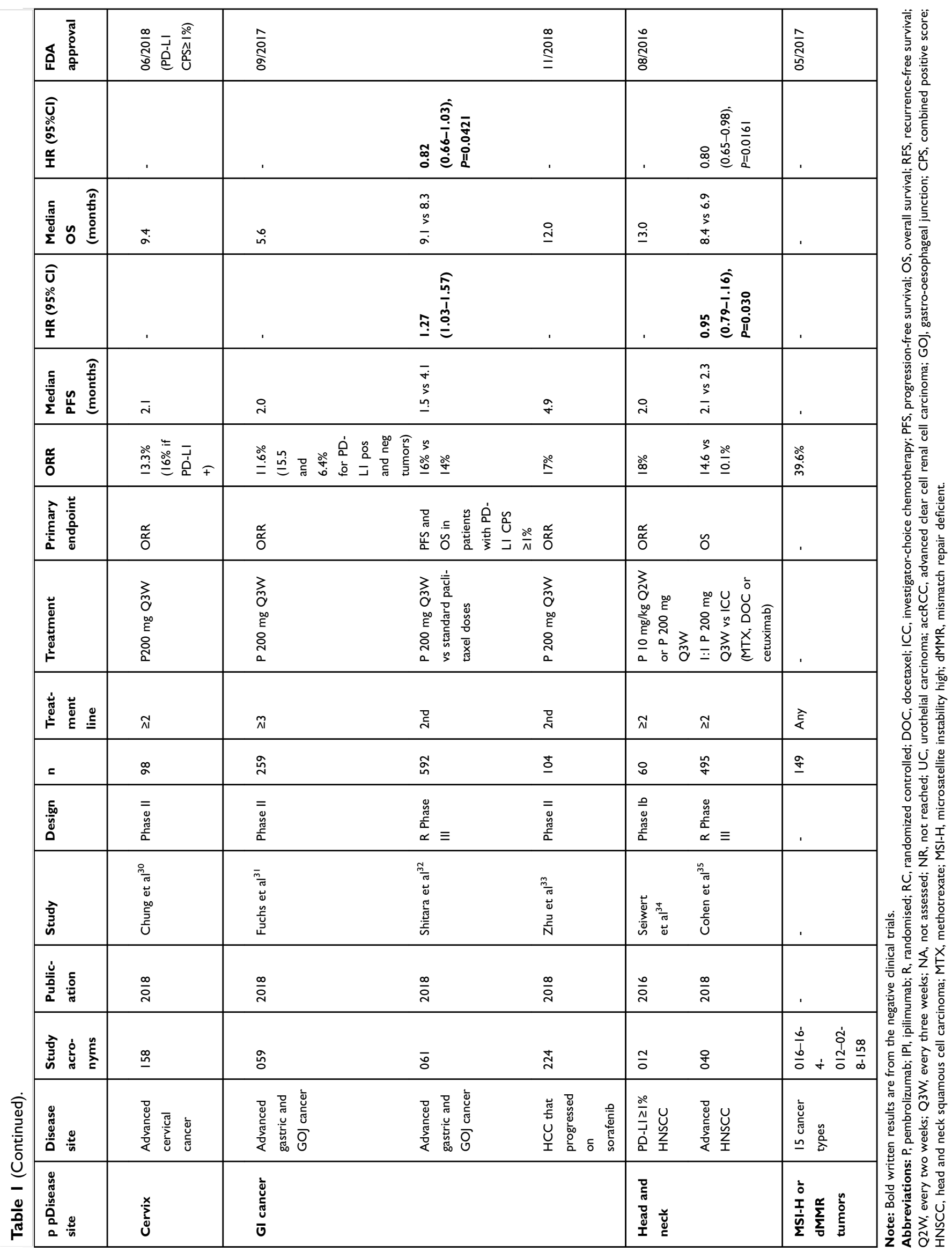




\section{Hepatocellular carcinoma (HCC)}

Pembrolizumab is approved as monotherapy in second-line of HCC based on the results of KEYNOTE-224 trial. This phase II trial included 104 HCC pretreated with sorafenib: ${ }^{33}$ ORR was $17 \%$. Of note, $25 \%$ of the patients were positive for hepatitis $\mathrm{B}$ and $\mathrm{C}$ virus, and an immune-related hepatitis occurred in three patients without viral flares. The ongoing KEYNOTE-240 trial is a phase III study aims at confirming these results.

\section{f. Head and neck}

The FDA approved pembrolizumab for recurrent or metastatic HNSCC on August 5, 2016. ${ }^{42}$ The KEYNOTE-012 is the first study investigating a PD-1 antibody for recurrent or metastatic HNSCC who progressed after platinum-containing chemotherapy. ${ }^{34-44}$ ORR was $18 \%$ including $4 \%$ of CR and $a \geq 6$ months DOR in $85 \%$ of the patients. The randomized phase III trial KEYNOTE-048 evaluated pembrolizumab monotherapy or combined with platinum/5FU chemotherapy vs the EXTREME regimen as first-line fin 882 PD-L1 CPS $\geq 20 \%$ HNSCC. ${ }^{45}$ Pembrolizumab alone or with chemotherapy significantly improved OS supporting pembrolizumab or pembrolizumab + platinum/5-FU as a new first-line standards of care for recurrent or metastatic HNSCC.

\section{g. MSI-H or dMMR tumors}

Five similar trials included 149 patients with various tumor types characterized by microsatellite instability-high (MSI-H) high or mismatch repair deficient (dMMR) (KEYNOTE-016 $(\mathrm{n}=58), \quad$ KEYNOTE-164 $(\mathrm{n}=61)$, KEYNOTE-012 $(\mathrm{n}=6)$, KEYNOTE-028 $(\mathrm{n}=5)$, and KEYNOTE-158 $(\mathrm{n}=19))$. The ORR with pembrolizumab across studies was $39.6 \%$ with $78 \%$ of responses lasting $\geq 6$ months. These innovative data led to an FDA granted accelerated approval for pembrolizumab in MSI-H or dMMR solid tumors progressing on treatment and without satisfactory alternative treatment options. This is the first time that a drug has a tissue-agnostic market authorization.

\section{Promising therapeutic trials of pembrolizumab}

Here are detailed the most promising trials of pembrolizumab as a monotherapy (Table 2):

\section{a. Lung and chest cancer}

\section{NSCLC}

KEYNOTE-091 aims at investigating the impact on outcome of adjuvant pembrolizumab after completion of radical surgery and standard adjuvant chemotherapy in the PD-L1+ subgroup and overall population. ${ }^{46}$ Primary results are awaited for August 19, 2021.

\section{SCC}

One complete response (CR) and 8 partial responses (PR) were observed in PD-L1 TPS $\geq 1 \%$ small cell carcinoma (SCC) in the multicohort phase $\mathrm{Ib}$ open-label KEYNOTE-028 trial. ${ }^{47,48}$ In the KEYNOTE-158, a phase II basket study, ${ }^{49}$ out of the $107 \mathrm{SCC}$, RR was $35.7 \%$ in PD-L1+ tumors vs $6.0 \%$ in PD-L1- tumors. The KEYNOTE-604 randomizes pembrolizumab/placebo with chemotherapy for newly diagnosed advanced SCC. ${ }^{50}$ Maintenance therapy with pembrolizumab was evaluated in 45 patients with advanced SCC following 4-6 cycles of platinum/etoposide. ${ }^{51}$ The disease control rate was $42 \%$ ( $1 \mathrm{CR}, 3 \mathrm{PR}$, and $15 \mathrm{SD})$ and median PFS and OS were 1.4 months and 9.2 months, respectively. In this small cohort, pembrolizumab did not improve PFS but improved OS suggesting that some patients might benefit from this strategy.

\section{Malignant pleural mesothelioma (MPM)}

PD-L1 is expressed in $20-40 \%$ and is a factor of worse prognosis. ${ }^{52,53}$ Pembrolizumab monotherapy provide a $20 \%$ ORR in PDL $1+$ TPS $\geq 1 \%$ MPM. $^{54}$ A Phase II trial of pembrolizumab is ongoing (NCT02399371). Preliminary results showed a response rate of $21 \%$ and a disease control rate of $76 \%$ and the optimization of PDL1 threshold is ongoing. ${ }^{55}$

\section{TC}

Thymic carcinomas and thymomas are rare tumors with limited therapeutic options. Two phase II (NCT02607631 and NCT02364076) showed an interesting ORR (24.2\% and $22.5 \%$ ) with pembrolizumab in that context. ${ }^{56,57}$

\section{b. Urothelial carcinoma Bladder}

Neoadjuvant pembrolizumab is evaluated in the PURE-01 phase 2 trial (NCT02736266). Cisplatin-eligible or ineligible patients will receive three cycles of the drug before surgery and radiologically non-responders are given three additional courses of dose-dense MVAC chemotherapy. Pathologic complete response (pT0) is the primary endpoint. The first 25 evaluable patients have been presented with a very satisfactory histological response rate of $8 / 25$ pT0 (32\%) and $3 / 25$ pTa/is. ${ }^{58}$ The ongoing KEYNOTE361 is a randomized, open-label, phase 3 study of 
Table 2 Promising therapeutic trials of pembrolizumab

\begin{tabular}{|c|c|c|c|c|c|}
\hline Disease site & Disease site & $\begin{array}{l}\text { Study } \\
\text { acro- } \\
\text { nyms }\end{array}$ & Design & $\begin{array}{l}\text { Treatment } \\
\text { line }\end{array}$ & Treatment \\
\hline \multirow[t]{2}{*}{ Lung } & SCC & 604 & RC Phase III & Ist & $\mathrm{CT}+\mathrm{P}$ or placebo \\
\hline & Stage IB-IIIA NSCLC & $\begin{array}{l}091 \\
\text { (PEARLS) }\end{array}$ & RC Phase III & Adjuvant & $\mathrm{CT}$ followed by $\mathrm{P}$ or placebo \\
\hline \multirow[t]{4}{*}{ Urothelial carcinomas } & Bladder & PURE-0I & Phase II & Neoadjuvant & $\begin{array}{l}3 \text { cycles of P } 200 \mathrm{mg} \mathrm{Q} 3 \mathrm{~W} \text { before } \\
\text { surgery }\end{array}$ \\
\hline & $\begin{array}{l}\text { Cisplatin eligible or ineligi- } \\
\text { ble advanced UC }\end{array}$ & 361 & R Phase III & Ist & $\mathrm{P}$ with or without $\mathrm{CT}$ vs $\mathrm{CT}$ alone \\
\hline & Kidney cancer & 427 & Phase II & Ist & $P$ \\
\hline & Kidney cancer & 426 & RC Phase III & Ist & $P+$ axitinib vs sunitinib \\
\hline breast & TNBC & 522 & & Neoadjuvant & $\begin{array}{l}\mathrm{CT} \pm \mathrm{P} \text { followed by } \mathrm{P} \text { or placebo } \\
\text { as maintenance therapy }\end{array}$ \\
\hline \multirow[t]{2}{*}{ CCR } & $\begin{array}{l}\text { MSI-high or MMR-deficient } \\
\text { CCR }\end{array}$ & 164 & Phase II & $\geq 2$ & $P$ \\
\hline & $\begin{array}{l}\text { MSI-high or MMR-deficient } \\
\text { CCR }\end{array}$ & 177 & RC Phase III & Ist & P vs SOC chemotherapy \\
\hline \multirow[t]{3}{*}{$\begin{array}{l}\text { Esophageal, GOJ and } \\
\text { gastric carcinoma }\end{array}$} & $\begin{array}{l}\text { Esophageal or GOJ } \\
\text { carcinoma }\end{array}$ & 181 & R phase III & 2nd & $P$ vs ICC \\
\hline & $\begin{array}{l}\text { Advanced or metastatic } \\
\text { esophageal carcinoma }\end{array}$ & 590 & RC phase III & Ist & Cisplatin $+5 \mathrm{FU}+\mathrm{P}$ or placebo \\
\hline & $\begin{array}{l}\text { PD-LI+, HER2- gastric or } \\
\text { GOJ carcinoma }\end{array}$ & 062 & R phase III & Ist & $\mathrm{P}$ vs $\mathrm{P}+\mathrm{CT}$ (cisplatin $+5 \mathrm{FU})$ \\
\hline
\end{tabular}

Abbreviations: P, pembrolizumab; R, randomised; RC, randomised controlled; CT, chemotherapy; ICC, investigator-choice chemotherapy; SOC, standard of care; PFS, progression-free survival; OS, overall survival; RFS, recurrence-free survival; Q2W, every two weeks; Q3W, every three weeks; UC, urothelial carcinoma; accRCC, advanced clear cell renal cell carcinoma; SCC, small cell carcinoma; NSCLC, non-small cell lung cancer; UC, urothelial carcinoma; TNBC, triple negative breast cancer; MSI, microsatellite instability; MMR, mismatch repair; CCR colorectal cancer; GOJ, gastro-oesophageal junction; HCC, hepatocellular carcinomas.

pembrolizumab \pm chemotherapy vs chemotherapy alone in both cisplatinum-eligible or ineligible patients with advanced untreated urothelial carcinoma.

\section{Kidney cancer}

KEYNOTE-427 evaluated pembrolizumab monotherapy as first-line in advanced clear cell (accRCC) and not clear cell RCC. The results of the accRCC cohort showed an ORR of $38.2 \%$ with long-lasting responses in $75 \%$ of the patients. $^{29}$ The promising KEYNOTE-426 study is an ongoing phase III multicenter, open-label, randomized trial designed to evaluate the efficacy and safety of pembrolizumab plus axitinib vs sunitinib alone in untreated metastatic RCC. 59

\section{Prostate cancer}

The KEYNOTE-199 trial is a 5-cohort phase II trial studying pembrolizumab monotherapy in metastatic pretreated CRPC. ${ }^{60}$ Anti-tumor activity was observed in all cohorts: disease control rate (DCR) was $26 \%$ (95\% CI 21 to 32 ) with $11 \%$ of the patients presenting DCR lasting more than 6 months.

\section{c. Breast cancer Neoadjuvant setting}

Pembrolizumab combined with chemotherapy provided up to $80 \%$ pathological response (ypT0 ypN0) in triple negative breast cancer (TNBC) in the KEYNOTE-173 trial. $^{61}$ The I-SPY2 phase II study assessed the addition of pembrolizumab to neoadjuvant paclitaxel followed by doxorubicin + cyclophosphamide for $\geq \mathrm{T} 2$ HER2 negative BC. ${ }^{62}$ Pembrolizumab improved pCR rates in all HER2 subtypes (pCR rate $46 \%$ vs $16 \%$ ), especially in TNBC. The KEYNOTE-522 is currently evaluating pembrolizumab + neoadjuvant chemotherapy followed by adjuvant pembrolizumab in TNBC. ${ }^{63}$ 


\section{TNBC}

Atezolizumab, an anti-PD-L1 antibody has shown a very interesting PFS benefit in front line therapy for TNBC in combination with nab-paclitaxel. ${ }^{64}$ The Keynote- 355 has randomized pembrolizumab/placebo with various regiments of chemotherapy (nab-paclitaxel, paclitaxel, or carboplatin/gemcitabine) in the same setting. ${ }^{65}$ The principal objectives are PFS and OS in all patients and in patients with PD-L1-positive tumors defined as PD-L1 staining in $\geq 1 \%$ tumor cells or in stroma. The results are warmly awaited in the next 6 months.

\section{HER2 positive BC}

The PANACEA Study evaluated pembrolizumab in combination with trastuzumab in patients with trastuzumabresistant, HER2+, PD-L1-positive (phase Ib) or negative (phase II) metastatic breast cancer. ${ }^{66}$ For PD-L1+ patients $(n=40)$ ORR and DCR were $15 \%$ and $25 \%$, respectively. No objective responses were observed in the PD-L1 negative cohort $(n=12)$.

\section{d. Gynecological cancer Endometrial cancer}

POLE (polymerase $\varepsilon$ ) mutated (6-12\%) and MSI endometrial tumors exhibited significantly elevated TILs, high expression of PD-1 and PD-L1 and greater peritumoral T-lymphocytes supporting trials of immune-checkpoint inhibitors. ${ }^{67,68}$ An ongoing single-institution phase II study of pembrolizumab in MMR deficient cancers included 9 patients with endometrioid carcinoma and showed an ORR of $56 \%$, including $1 \mathrm{CR}^{69}$ The KEYNOTE-775 is currently evaluating pembrolizumab plus lenvatinib vs chemotherapy in second line endometrial cancer (NCT03517449).

\section{Ovarian cancer}

KEYNOTE-100 is an ongoing phase II for relapsing ovarian cancer after front-line platinum-based therapy. ${ }^{70}$ Of the 376 patients included, ORR was 9\%, median PFS was 2.1 months. ORR reaches $14 \%$ and $25 \%$ for patients with PDL1 CPS $\geq 1 \%$ and $\geq 10 \%$. Homologous recombination deficiencies are a frequent hallmark of serous high-grade ovarian cancer leading to the approval of PARP inhibitors. The combination of PARPi with immunotherapy seems very promising by boosting the immune response. TOPACIO/KEYNOTE-162 phase I/II study evaluated the association of Niraparib and pembrolizumab in platinumresistant ovarian cancer and TNBC. ${ }^{71}$ ORR and DCR were $25 \%$ and $68 \%$ and $45 \%$ and $73 \%$ in the 11 BRCA-mutated patients. NEOPEMBROV is an ongoing randomized, controlled phase II study for ovarian cancers that are not eligible for primary surgery. Patients will receive pembrolizumab or placebo added to with carboplatin and paclitaxel. Primary objective is to evaluate the complete resection rate after interval debulking surgery.

\section{e. Gastrointestinal cancer CRC}

The phase II KEYNOTE-016 was conducted to evaluate pembrolizumab in metastatic CRC with (MMR deficient) or without (MMR proficient) MMR deficiency: ${ }^{72,73}$ The ORR was much higher in MMRd tumor: $40 \%$ vs $0 \%$, as was PFS rate: 78 vs $11 \%$ for MMR deficient and proficient patients. The KEYNOTE-164 aims at confirming these data by recruiting MSI-H CCR defined by PCR-based assay or lack of expression of $\geq 1$ MMR protein (MLH1, MSH2, MSH6, PMS2) by IHC. ${ }^{74}$ The KEYNOTE-177 is an international, randomized, open-label, phase 3 study of pembrolizumab vs standard-of-care chemotherapy in firstline MMR-deficient or MSI-high metastatic CRC. ${ }^{75}$

\section{Esophageal cancer}

The phase II KEYNOTE-180 will evaluate pembrolizumab as a monotherapy in patients with previously treated advanced or metastatic esophageal cancer. ${ }^{76}$ KEYNOTE181 is a phase III trial comparing pembrolizumab vs standard therapy in advanced esophageal or gastroesophageal junction carcinoma that progressed after firstline therapy. ${ }^{77}$ KEYNOTE-590 is designed to evaluate efficacy and safety of pembrolizumab vs placebo plus cisplatin and 5-FU chemotherapy as first-line treatment in participants with locally advanced or metastatic esophageal carcinoma.

\section{Squamous cell carcinoma of the anal canal (SCCA)} KEYNOTE-028 included PD-L1+ SCCA. PD-L1 positivity was found in $74 \%$ of the screened patients. Among the 24 patients, no CR were noted but 4 patients had a PR, for an ORR of $17 \%$, and 10 patients $(42 \%)$ had a confirmed stable disease. The DCR was $58 \%{ }^{78}$ A phase II study in refractory metastatic SCCA is currently recruiting (NCT02919969).

\section{Biliary tract}

PD-L1 is highly expressed in cholangiocarcinoma in association to a high density of CD3-positive tumor-infiltrating lymphocytes suggesting a potential role for pembrolizumab. ${ }^{79}$ KEYNOTE-028 basket trial enrolled 24 patients with PD-L1+ biliary tract cancer to receive pembrolizumab monotherapy. ${ }^{80}$ Four PR have been 
observed prompting a successor biliary cancer cohort of 100 patients in the ongoing KEYNOTE-158 basket trial (NCT02628067). Not yet recruiting, the NCT03260712 will evaluate Cisplatin + Gemcitabine and pembrolizumab in that context.

\section{f. Head and neck}

The KEYNOTE-055 phase II study included 171 patients with HNSCC progressing within 6 months of platinum-based chemotherapy with cetuximab. ${ }^{81} 82 \%$ of the patients were PD-L1 positive and $22 \%$ were HPV positive. ORR with pembrolizumab monotherapy was $16 \%$ with a median DOR of 8 months (range: 2 to 12). Response rates were similar in all HPV and PD-L1 subgroups. Median PFS and OS were 2.1 and 8 months, respectively. The KEYNOTE-040 was a randomized phase III study which included patients with HNSCC after a platinumbased chemotherapy to receive either pembrolizumab or standard of care. ${ }^{35}$ Primary endpoint (PFS and OS) was not reached. Median OS was not statistically higher with pembrolizumab. Subgroups analyses showed a weighty prolonged OS proportionally to PD-L1 expression: OS was 8.7 months $(\mathrm{HR}=0.75(95 \% \mathrm{CI} 0.59$ to 0.95$), P=0.0078)$ for $\mathrm{PD}-\mathrm{L} 1$ $\mathrm{CPS} \geq 1 \%$; and OS was 11.6 vs 7.9 months $(\mathrm{HR}=0.54(95 \%$ CI 0.35 to 0.82 ), $P=0.0017$ ) for PD-L1 TPS $\geq 50 \%$.

The following ongoing studies will evaluate pembrolizumab for high-risk HNSCC in both neoadjuvant and adjuvant setting (phase II, NCT02641093; phase II for HPV negative HNSCC NCT02296684). ${ }^{82}$ KEYNOTE412 will evaluate the addition of pembrolizumab in combination with concomitant chemo-radiotherapy for locally advanced HNSCC. ${ }^{83}$

\section{g. Rare tumors}

ACSé pembrolizumab (NCT03012620) is a multi-cohort phase II study designed to propose secured accessed to pembrolizumab for patients with rare cancer. Seven cohorts are recruiting including rare sarcoma, rare ovarian cancer, primary central nervous system lymphomas, rare thyroid cancer, rare malignant neuroendocrine cancer, germ-cell cancer, and NK/T-cell lymphoma.

\section{Predictive factors of response to pembrolizumab and selection of patients}

\section{a. PD-LI status}

PD-L1 expression is the main predictive biomarker of response, and its expression is often associated with a poor prognosis. Some important questions are still unsolved. Whether the PD-L1 positivity must be determined on tumor cell, immune cell or both is unknown. Therefore, PD-L1 positivity definitions vary across studies with scores like tumor proportion score (TPS) or combined proportion score (CPS) which is the sum of the percentage of PD-L1 expressing tumor cells and immune cells as a fraction of the number of tumor cells. ${ }^{84}$ The optimal threshold of PD-L1 positivity to select the patients is not established varying from $1 \%$ to $50 \%$, for example, in NSCLC and different antibodies are used. In addition, some variations of PD-L1 expression have been observed between the course of the disease raising the question of the best moment to evaluate PD-L1 expression (primitive tumor vs metastases). ${ }^{85,86}$ Lastly, complete response are observed in $17 \%$ of the PD-L1-negative advanced melanoma. ${ }^{87}$ PD-L1 positivity is important but not sufficient for identify responders.

\section{b. Mutational load and neoantigen burden} The tumor mutational load (TML) corresponds to the somatic mutation rate for a given tumor and varies according to the tissue of origin of cancer. ${ }^{88}$ A high TML leads to the expression of multiple neoantigens and triggers an immune response. Therefore, a high TML is potentially associated with the efficacy of checkpoint inhibitors. These observations were validated in two cohorts of lung cancer receiving pembrolizumab where a high TML was associated with ORR, durable clinical benefit, and PFS. ${ }^{89}$ A retrospective study of various tumor types showed that mutational load and T-cell-inflamed microenvironment were predictors of response to pembrolizumab. ${ }^{90}$ Median number of mutations was 180 in responders vs 61 in nonresponders. Another retrospective study including various tumor types demonstrated that a threshold of $\geq 20$ mutations/megabase was associated with improved ORR, PFS, and OS . ${ }^{91}$ Prospective study data are expected to validate the value of TMB.

\section{c. Mismatch repair deficiency}

An analysis of tumor mutational load in 100,000 cancer genomes identified a novel mutation hotspot in the promoter of the DNA mismatch repair gene PMS2, that was significantly associated with high tumor mutational load. $^{92}$ Whole-exome sequencing also showed that MMR deficient tumors are largely most mutated than MMR proficient tumors (1782 vs 73 somatic mutations per tumors, $P=0.007$ ) associated with prolonged PFS 
for high somatic mutational load. ${ }^{72}$ This high tumor mutation load is also associated with genetic alterations leading to dysregulation of the mechanisms of DNA repair, such as microsatellite instability and the POLE gene, as shown in endometrial cancer. In the phase II KEYNOTE-016 which includes MMR proficient and deficient colorectal cancer patient and MMR deficient cancer that were not colorectal, MMR deficiency predicts response of solid tumors to PD-1 blockade by pembrolizumab. ${ }^{73,93}$ Four more clinical trials included patients with different cancers with the common characteristic of MMR deficiency, concluding that pembrolizumab was effective in this population. These data, which led to the FDA approval, however, remain preliminary, and the results of the phase 3 studies are firmly awaited.

\section{d. Need of new response criteria?}

Response Evaluation Criteria in Solid Tumours 1.1 (RECIST) criteria using CT (computed tomography) scan are the gold standards to evaluate the efficacy of drugs. Efforts have been made with the development of irRC (immune-related Response Criteria), irRECIST (immunerelated RECIST), and iRECIST (immune RECIST) without changing the standards of care for the moment. IrRECIST and iRECIST seem to better identify patients with unconventional response as false progressors. ${ }^{94}$ Metabolic imaging like positron emission tomography (PET) tracers as 18 F-fluorodeoxyglucose (FDG) are known to be taken up in inflammatory cells, what may predict response to immunotherapy better than $\mathrm{CT} .{ }^{95-97}$

\section{How to increase the efficacy of pembrolizumab?}

\section{a. To warm up the tumor microenvironment}

Tumors are now considered like a continuum between hot and cold tumors, depending on the density of infiltration by immune cells. Tumor inflammation composite scores have been evaluated and indicate that the efficacy of pembrolizumab is correlated to a high density of immune cells. ${ }^{34,98,99}$ Different strategies aiming at increasing infiltration of immune cell in tumor site are currently evaluated.

Patients who do not respond to anti-PD-1 antibody lack CD8+ T cells inside the tumor lesions.100 A phase Ib study (MASTERKEY-265) combining pembrolizumab with an oncolytic virotherapy (talimogene laherparepvec (TVEC)) administered in the tumor advanced melanoma. ${ }^{101,102}$ ORR rate was $62 \%$, with a complete response rate of $33 \%$ per irRC. CD8+ T cells level, elevated PD-L1 expression, and IFN-g gene expression were observed in tumors of responders also in distant lesions. The phase III trial is ongoing in melanoma. Another strategy of combination of pembrolizumab with antiTLR9 (a stimulating agent tumor microenvironment by activating plasmacytoid dendritic cells) is ongoing. CMP-001 comprises a CpG-A oligodeoxynucleotide packaged within a virus-like particle. CMP-001-001 is an ongoing phase $\mathrm{Ib}$ trial evaluating intratumoral (IT) CMP-001 in combination with pembrolizumab in PD-1 resistant advanced melanoma (either did not respond or progressed) on prior anti-PD-1 monotherapy or in combination. ${ }^{103, p 146}$ Safety data of 63 patients demonstrated a manageable toxicity with fever, N/V, headache, hypotension, and rigors. Grade 3/4-related AEs were reported in 15 of 68 patients. The ORR across all dose cohorts on weekly $(\mathrm{n}=40)$ and Q3W schedules $(\mathrm{n}=13)$ were $22.5 \%$ ( $9 / 40 ; 95 \%$ CI $11 \%$ to $39 \%)$ and $7.7 \% \%(1 / 13 ; 95 \%$ CI $0 \%$ to $36 \%$ ) respectively. Distant regression of non-injected tumors occurred in cutaneous, nodal, hepatic, and splenic metastases. CMP-001 dosing at $5 \mathrm{mg}$ /weekly has been selected for further evaluation in the ongoing dose expansion phase of this study. Epacadostat is an indoleamine-2,3-dioxygenase 1 (IDO1) inhibitor. IDO1 is an endogenous mechanism of acquired peripheral immune tolerance in vivo. ${ }^{104}$ Several therapeutic trials are evaluating epacadostat with pembrolizumab. Two ongoing phase III studies are testing the combination in UC, after first-line platinum, ${ }^{105}$ or for cisplatinum-ineligible patients 106 In head and neck SCC, the phase I/II study ECHO202/KEYNOTE-037 also have shown promising results with an ORR for patients with 1-2 or 3 prior line of $34 \%$ and $14 \%$, respectively. ${ }^{107}$ An ongoing phase III study randomizes pembrolizumab plus epacadostat vs pembrolizumab and vs the EXTREME regimen as first-line treatment for advanced head and neck squamous cell carcinoma (ECHO-304/KEYNOTE669). ${ }^{108}$ However a phase III in melanoma failed to increase PFS compared to pembrolizumab monotherapy. ${ }^{109}$

\section{b. Combination with other checkpoint inhibitor}

Although pembrolizumab has supplanted ipilimumab in the management of melanoma, it was the first checkpoint inhibitor to demonstrate its efficacy in melanoma. The KEYNOTE-029, ${ }^{110}$ an open-label phase Ib study tested pembrolizumab $2 \mathrm{mg} / \mathrm{kg}$ plus ipilimumab $1 \mathrm{mg} / \mathrm{kg}$ every 3 weeks for four doses, followed by pembrolizumab 
monotherapy. Grade 3-4 treatment-related AE were particularly high (45\%) with an ORR of $60 \%$.

\section{c. Association of pembrolizumab with targeted therapies}

Multiple studies are currently testing pembrolizumab in combination with already approved therapies. Lenvatinib is a multikinase inhibitor of vascular endothelial growth factor receptor 1-3, fibroblast growth factor receptor 1-4, plateletderived growth factor receptor $\alpha$, RET, and KIT. In 13 patients with unresectable HCC, ${ }^{111}$ the combination pembrolizumab/ lenvatinib provided an ORR of $46 \%$. In 22 HNSCC regardless of PD-L1 status, this combination provided consistent and durable responses were seen: ORR (36.4\%), median DOR (8.2 months), and PFS (8.2 months). ${ }^{112}$ Vorinostat, an HDAC inhibitor has been combined with pembrolizumab in recurrent metastatic HNSCC and salivary gland cancer with an ORR of $36 \%$ and $16 \%$, respectively. ${ }^{113}$

\section{Conclusion}

The role of pembrolizumab in the management of cancer is no longer to prove. Indeed, many marketing authorizations have been granted to pembrolizumab by the FDA in various types of cancer. Better tolerated and more effective than conventional treatments, this makes it a treatment of choice. Selecting the patients benefiting at best from the therapy remains challenging. PD-L1 expression, the most used biomarker, remains imperfect. Numerous combination trials are ongoing.

\section{Disclosure}

Marie Robert received travel expenses. Marie Robert also reports honoraria from Merck and Novartis during the conduct of the study. Mario Campone acted in an advisory role for Novartis, Sanofi, Pierre Fabre, Lilly and Astra Zeneca outside the submitted work. The other authors report no conflicts of interest in this work.

\section{References}

1. Scapin G, Yang X, Prosise WW, et al. Structure of full-length human anti-PD1 therapeutic IgG4 antibody pembrolizumab. Nat Struct Mol Biol. 2015;22(12):953-958. doi:10.1038/nsmb.3129

2. Blank C, Brown I, Peterson AC, et al. PD-L1/B7H-1 inhibits the effector phase of tumor rejection by t cell receptor (TCR) transgenic $\mathrm{CD} 8^{+} \mathrm{T}$ cells. Cancer Res. 2004;64(3):1140-1145. doi:10.1158/0008-5472.CAN-03-3259

3. Okazaki T, Honjo T. PD-1 and PD-1 ligands: from discovery to clinical application. Int Immunol. 2007;19(7):813-824. doi:10.1093/intimm/dxm057

4. Sharp M, Corp D, Kenilworth NJ: KEYTRUDA ${ }^{\circledR}$ (pembrolizumab) Prescribing Information.pdf. Available from: http://www.merck.com/pro duct/usa/pi_circulars/k/keytruda/keytruda_pi.pdf. Accessed October 6, 2018.
5. Ahamadi M, Freshwater T, Prohn M, et al. model-based characterization of the pharmacokinetics of pembrolizumab: a humanized anti-pd1 monoclonal antibody in advanced solid tumors: pharmacokinetics of pembro in solid tumors. CPT Pharmacometrics Syst Pharmacol. 2017;6(1):49-57. doi:10.1002/psp4.12139

6. Longoria TC, Tewari KS. Evaluation of the pharmacokinetics and metabolism of pembrolizumab in the treatment of melanoma. Expert Opin Drug Metab Toxicol. 2016;12(10):1247-1253. doi: $10.1080 / 17425255.2016 .1216976$

7. Garon EB, Rizvi NA, Hui R, et al. Pembrolizumab for the treatment of non-small-cell lung cancer. $N$ Engl J Med. 2015;372 (21):2018-2028. doi:10.1056/NEJMoa1501824

8. Zhang T, Xie J, Arai S, et al. The efficacy and safety of anti-PD-1/PDL1 antibodies for treatment of advanced or refractory cancers: a meta-analysis. Oncotarget. 2016;7:45. doi:10.18632/oncotarget.12230

9. Wang M, Ma X, Guo L, Xia F. Safety and efficacy profile of pembrolizumab in solid cancer: pooled reanalysis based on randomized controlled trials. Drug Des Devel Ther. 2017;11:2851-2860. doi:10.2147/DDDT.S146286

10. Sznol M, Postow MA, Davies MJ, et al. Endocrine-related adverse events associated with immune checkpoint blockade and expert insights on their management. Cancer Treat Rev. 2017;58:70-76. doi:10.1016/j.ctrv.2017.06.002

11. Michot JM, Bigenwald C, Champiat S, et al. Immune-related adverse events with immune checkpoint blockade: a comprehensive review. Eur J Cancer. 2016;54:139-148. doi:10.1016/j.ejca.2015.11.016

12. Postow MA, Callahan MK, Wolchok JD. Immune checkpoint blockade in cancer therapy. $J$ Clin Oncol. 2015;33 (17):1974-1982. doi:10.1200/JCO.2014.59.4358

13. Feng S, Coward J, McCaffrey E, Coucher J, Kalokerinos P, O'Byrne K. Pembrolizumab-induced encephalopathy: a review of neurological toxicities with immune checkpoint inhibitors. $J$ Thorac Oncol. 2017;12(11):1626-1635. doi:10.1016/j. jtho.2017.08.007

14. Leroy V, Templier C, Faivre J-B, et al. Pembrolizumab-induced pneumonitis. ERJ Open Res. 2017;3(2):00081-02016. doi:10.1183/ 23120541.00081-2016

15. Escandon J, Peacock S, Trabolsi A, Thomas DB, Layka A, Lutzky J. Interstitial nephritis in melanoma patients secondary to PD-1 checkpoint inhibitor. J Immunother Cancer. 2017;5:1. doi:10.1186/s40425-016-0205-2

16. Wu Z, Lai L, Li M, Zhang L, Zhang W. Acute liver failure caused by pembrolizumab in a patient with pulmonary metastatic liver cancer: A case report. Medicine (Baltimore). 2017;96(51):e9431. doi:10.1097/MD.0000000000009431

17. Läubli H, Balmelli C, Bossard M, Pfister O, Glatz K, Zippelius A. Acute heart failure due to autoimmune myocarditis under pembrolizumab treatment for metastatic melanoma. $J$ Immunother Cancer. 2015;3:1. doi:10.1186/s40425-015-0057-1

18. Geukes Foppen MH, Rozeman EA, van Wilpe S, et al. Immune checkpoint inhibition-related colitis: symptoms, endoscopic features, histology and response to management. ESMO Open. 2018;3(1):e000278. doi:10.1136/esmoopen-2017-000278

19. Robert C, Ribas A, Wolchok JD, et al. Anti-programmed-deathreceptor-1 treatment with pembrolizumab in ipilimumab-refractory advanced melanoma: a randomised dose-comparison cohort of a phase 1 trial. The Lancet. 2014;384(9948):1109-1117. doi:10.1016/S0140-6736(14)60958-2

20. Ribas A, Puzanov I, Dummer R, et al. Pembrolizumab versus investigator-choice chemotherapy for ipilimumab-refractory melanoma (KEYNOTE-002): a randomised, controlled, phase 2 trial. Lancet Oncol. 2015;16(8):908-918. doi:10.1016/S1470-2045(15) 00083-2

21. Robert C, Schachter J, Long GV, et al. Pembrolizumab versus Ipilimumab in Advanced Melanoma. $N$ Engl J Med. 2015;372 (26):2521-2532. doi:10.1056/NEJMoa1503093 
22. Herbst RS, Baas P, Kim D-W, et al. Pembrolizumab versus docetaxel for previously treated, PD-L1-positive, advanced non-smallcell lung cancer (KEYNOTE-010): a randomised controlled trial. The Lancet. 2016;387(10027):1540-1550. doi:10.1016/S01406736(15)01281-7

23. Reck M, Rodríguez-Abreu D, Robinson AG, et al. Pembrolizumab versus chemotherapy for PD-L1-positive non-small-cell lung cancer $N$ Engl J Med. 2016;375(19):1823-1833. doi:10.1056/ NEJMoa1606774

24. Lopes G, Wu Y-L, Kudaba I, et al. Pembrolizumab (pembro) versus platinum-based chemotherapy (chemo) as first-line therapy for advanced/metastatic NSCLC with a PD-L1 tumor proportion score (TPS) $\geq 1 \%$ : open-label, phase 3 KEYNOTE-042 study. $J$ Clin Oncol. 2018;36(18_suppl):LBA4-LBA4. doi:10.1200/ JCO.2018.36.18_suppl.LBA4

25. Gandhi L, Rodríguez-Abreu D, Gadgeel S, et al. Pembrolizumab plus chemotherapy in metastatic non-small-cell lung cancer. $N$ Engl J Med 2018;378(22):2078-2092. doi:10.1056/NEJMoa1801005

26. Paz-Ares LG, Luft A, Tafreshi A, et al. Phase 3 study of carboplatin-paclitaxel/nab-paclitaxel (Chemo) with or without pembrolizumab (Pembro) for patients (Pts) with metastatic squamous (Sq) non-small cell lung cancer (NSCLC). J Clin Oncol. 2018;36 (15_suppl):105. doi:10.1200/JCO.2018.36.15_suppl.105

27. Bellmunt J, de Wit R, Vaughn DJ, et al. Pembrolizumab as second-line therapy for advanced urothelial carcinoma. $N$ Engl $J$ Med. 2017;376(11):1015-1026. doi:10.1056/NEJMoa1613683

28. Balar AV, Castellano D, O'Donnell PH, et al. First-line pembrolizumab in cisplatin-ineligible patients with locally advanced and unresectable or metastatic urothelial cancer (KEYNOTE-052): a multicentre, single-arm, phase 2 study. Lancet Oncol. 2017;18(11):1483-1492. doi:10.1016/S14702045(17)30072-4

29. McDermott DF, Lee J-L, Szczylik C, et al. Pembrolizumab monotherapy as first-line therapy in advanced clear cell renal cell carcinoma (accRCC): results from cohort A of KEYNOTE-427. J Clin Oncol. 2018;36(15_suppl):4500. doi:10.1200/JCO.2018.36.15 suppl.4500

30. Chung HC, Schellens JHM, Delord J-P, et al. Pembrolizumab treatment of advanced cervical cancer: updated results from the phase 2 KEYNOTE-158 study. $J$ Clin Oncol. 2018;36 (15 suppl):5522. doi:10.1200/JCO.2018.36.15 suppl.5522

31. Fuchs CS, Doi T, Jang RW, et al. Safety and efficacy of pembrolizumab monotherapy in patients with previously treated advanced gastric and gastroesophageal junction cancer: phase 2 clinical KEYNOTE-059 trial. JAMA Oncol. 2018;4(5):e180013. 10.1001/ jamaoncol.2018.0013.

32. Shitara K, Özgüroğlu M, Bang Y-J, et al. Pembrolizumab versus paclitaxel for previously treated, advanced gastric or gastro-oesophageal junction cancer (KEYNOTE-061): a randomised, open-label, controlled, phase 3 trial. The Lancet. 2018. doi:10.1016/S0140-6736(18)31257-1

33. Zhu AX, Finn RS, Edeline J, et al. Pembrolizumab in patients with advanced hepatocellular carcinoma previously treated with sorafenib (KEYNOTE-224): a non-randomised, open-label phase 2 trial. Lancet Oncol. 2018;19(7):940-952. doi:10.1016/S1470-2045(18)30351-6

34. Seiwert TY, Burtness B, Mehra R, et al. Safety and clinical activity of pembrolizumab for treatment of recurrent or metastatic squamous cell carcinoma of the head and neck (KEYNOTE-012): an open-label, multicentre, phase $1 \mathrm{~b}$ trial. Lancet Oncol. 2016;17(7):956-965. doi:10.1016/S14702045(16)30066-3

35. Cohen EEW, Soulières D, Le Tourneau C, et al. Pembrolizumab versus methotrexate, docetaxel, or cetuximab for recurrent or metastatic head-and-neck squamous cell carcinoma (KEYNOTE-040): a randomised, open-label, phase 3 study. The Lancet. 2018. doi:10.1016/S0140-6736(18)31999-8
36. Eggermont AMM, Blank CU, Mandala M, et al. Adjuvant Pembrolizumab versus placebo in resected stage III melanoma. $N$ Engl J Med. 2018;378(19):1789-1801. doi:10.1056/ NEJMoa1802357

37. Mok T, Wu Y-L, Watson PA, Zhang J, Rangwala RA, Lopes G. Phase 3 KEYNOTE-042 trial of pembrolizumab (MK-3475) versus platinum doublet chemotherapy in treatment-naive patients (pts) with PD-L1-positive advanced non-small cell lung cancer (NSCLC). J Clin Oncol. 2015;33(15_suppl):TPS8105-TPS8105. doi:10.1200/jco.2015.33.15_suppl.tps8105

38. Vuky J, Balar AV, Castellano DE, et al. Updated efficacy and safety of KEYNOTE-052: A single-arm phase 2 study investigating first-line pembrolizumab (pembro) in cisplatin-ineligible advanced urothelial cancer (UC). J Clin Oncol. 2018;36(15_suppl):4524. doi:10.1200/JCO.2018.36.15_suppl.4524

39. Frenel J-S, Le Tourneau C, O'Neil B, et al. Safety and Efficacy of pembrolizumab in advanced, programmed death ligand 1-positive cervical cancer: results from the phase Ib KEYNOTE-028 trial. J Clin Oncol. 2017;35(36):4035-4041. doi:10.1200/JCO.2017.74.5471

40. Fashoyin-Aje L, Donoghue $\mathrm{M}$, Chen $\mathrm{H}$, et al. FDA approval summary: pembrolizumab for recurrent locally advanced or metastatic gastric or gastroesophageal junction adenocarcinoma expressing PD-L1. Oncologist. 2018. theoncologist.2018-0221. doi:10.1634/theoncologist.2018-0221

41. Tabernero J, Bang Y-J, Fuchs CS, et al. KEYNOTE-062: phase III study of pembrolizumab (MK-3475) alone or in combination with chemotherapy versus chemotherapy alone as first-line therapy for advanced gastric or gastroesophageal junction (GEJ) adenocarcinoma. $J$ Clin Oncol. 2016;34(4_suppl):TPS185TPS185. doi:10.1200/jco.2016.34.4_suppl.tps 185

42. Larkins E, Blumenthal GM, Yuan $\bar{W}$, et al. FDA approval summary: pembrolizumab for the treatment of recurrent or metastatic head and neck squamous cell carcinoma with disease progression on or after platinum-containing chemotherapy. Oncologist. 2017;22 (7):873-878. doi:10.1634/theoncologist.2016-0496

43. Chow LQM, Haddad R, Gupta S, et al. Antitumor activity of pembrolizumab in biomarker-unselected patients with recurrent and/or metastatic head and neck squamous cell carcinoma: results from the phase $\mathrm{Ib}$ KEYNOTE-012 expansion cohort. $J$ Clin Oncol. 2016;34 (32):3838-3845. doi:10.1200/JCO.2016.68.1478

44. Mehra R, Seiwert TY, Gupta S, et al. Efficacy and safety of pembrolizumab in recurrent/metastatic head and neck squamous cell carcinoma: pooled analyses after long-term follow-up in KEYNOTE-012. $\mathrm{Br}$ J Cancer. 2018. doi:10.1038/s41416-018-0131-9

45. Burtness B, Harrington KJ, Greil R, et al. KEYNOTE-048: phase III study of first-line pembrolizumab (P) for recurrent/metastatic head and neck squamous cell carcinoma (R/M HNSCC). Ann Oncol. 2018;29(suppl 8). doi:doi:10.1093/annonc/mdy424

46. O'Brien MER, Hasan B, Dafni U, et al. EORTC-ETOP randomized, phase 3 trial with anti-PD-1 monoclonal antibody pembrolizumab versus placebo for patients with early stage non-small cell lung cancer (NSCLC) after resection and standard adjuvant chemotherapy: PEARLS (NCT02504372). J Clin Oncol. 2016;34(15_suppl): TPS8571-TPS8571. doi:10.1200/JCO.2016.34.15_suppl.TPS8571

47. Ott PA, Elez E, Hiret S, et al. Pembrolizumab in patients with extensive-stage small-cell lung cancer: results from the phase Ib KEYNOTE-028 study. J Clin Oncol. 2017;35(34):3823-3829. doi: 10.1200/JCO.2017.72.5069

48. Ott P, Felip E, Hiret S, et al. OA05.01 pembrolizumab in patients with extensive-stage small cell lung cancer: updated survival results from KEYNOTE-028. J Thorac Oncol. 2017;12(1):S259. doi:10.1016/j.jtho.2016.11.250

49. Chung HC, Lopez-Martin JA, Kao SC-H, et al. Phase 2 study of pembrolizumab in advanced small-cell lung cancer (SCLC): KEYNOTE-158. $J$ Clin Oncol. 2018;36(15_suppl):8506. doi:10.1200/JCO.2018.36.15_suppl.8506 
50. Rudin C, Shen L, Pietanza MC. P2.04-007 KEYNOTE-604: phase 3 randomized, double-blind trial of pembrolizumab/placebo plus etoposide/platinum for extensive stage-SCLC. $J$ Thorac Oncol. 2017;12(11):S2400. doi:10.1016/j.jtho.2017.11.020

51. Gadgeel SM, Ventimiglia J, Kalemkerian GP, et al. Phase II study of maintenance pembrolizumab (pembro) in extensive stage small cell lung cancer (ES-SCLC) patients (pts). J Clin Oncol. 2017;35 (15_suppl):8504. doi:10.1200/JCO.2017.35.15_suppl.8504

52. Cedrés S, Ponce-Aix S, Zugazagoitia J, et al. Analysis of expression of programmed cell death 1 ligand 1 (PD-L1) in Malignant Pleural Mesothelioma (MPM). Gangopadhyay N, ed. PLoS One. 2015;10(3):e0121071. doi:10.1371/journal.pone.0121071

53. Mansfield AS, Roden AC, Peikert T, et al. B7-H1 expression in malignant pleural mesothelioma is associated with sarcomatoid histology and poor prognosis. $J$ Thorac Oncol. 2014;9 (7):1036-1040. doi:10.1097/JTO.0000000000000177

54. Alley EW, Lopez J, Santoro A, et al. Clinical safety and activity of pembrolizumab in patients with malignant pleural mesothelioma (KEYNOTE-028): preliminary results from a non-randomised, open-label, phase 1b trial. Lancet Oncol. 2017;18(5):623-630. doi:10.1016/S1470-2045(17)30169-9

55. Kindler H, Karrison T, Y-H CT, et al. OA13.02 phase II trial of pembrolizumab in patients with Malignant Mesothelioma (MM): interim analysis. $J$ Thorac Oncol. 2017;12(1):S293-S294. doi:10.1016/j.jtho.2016.11.301

56. Cho J, Ahn M-J, Yoo KH, et al. A phase II study of pembrolizumab for patients with previously treated advanced thymic epithelial tumor. J Clin Oncol. 2017;35(15_suppl):8521. doi:10.1200/ JCO.2017.35.15 suppl.8521

57. Giaccone G, Kim C, Thompson J, et al. Pembrolizumab in patients with thymic carcinoma: a single-arm, single-centre, phase 2 study. Lancet Oncol. 2018;19(3):347-355. doi:10.1016/S1470-2045(18) 30062-7

58. Necchi A, Briganti A, Raggi D, et al. Interim results from PURE-01: A phase 2, open-label study of neoadjuvant pembrolizumab (pembro) before radical cystectomy for muscle-invasive urothelial bladder carcinoma (MIUC). J Clin Oncol. 2018;36(6_suppl):TPS533-TPS533. doi:10.1200/JCO.2018.36.6_suppl.TPS533

59. Rini BI, Powles T, Chen M, Puhlmann M, Atkins MB. Phase 3 KEYNOTE-426 trial: pembrolizumab (pembro) plus axitinib versus sunitinib alone in treatment-naive advanced/metastatic renal cell carcinoma (mRCC). J Clin Oncol. 2017;35(15_suppl):TPS4597TPS4597. doi:10.1200/JCO.2017.35.15_suppl.TPS4597

60. De Bono JS, Goh JC, Ojamaa K, et al. KEYNOTE-199: pembrolizumab (pembro) for docetaxel-refractory metastatic castration-resistant prostate cancer (mCRPC). J Clin Oncol. 2018;36(15_suppl):5007. doi:10.1200/JCO.2018.36.15_suppl.5007

61. Schmid P, Park YH, Muñoz-Couselo E, et al. Pembrolizumab (pembro) + chemotherapy (chemo) as neoadjuvant treatment for triple negative breast cancer (TNBC): preliminary results from KEYNOTE-173. J Clin Oncol. 2017;35(15_suppl):556. doi:10.1200/JCO.2017.35.15_suppl.556

62. Nanda R, Liu MC, Yau C, et al. Pembrolizumab plus standard neoadjuvant therapy for high-risk breast cancer (BC): results from I-SPY 2. J Clin Oncol. 2017;35(15_suppl):506. doi:10.1200/ JCO.2017.35.15_suppl.506

63. Schmid P, Cortes J, Bergh JCS, et al. KEYNOTE-522: phase III study of pembrolizumab (pembro) + chemotherapy (chemo) vs placebo + chemo as neoadjuvant therapy followed by pembro vs placebo as adjuvant therapy for triple-negative breast cancer (TNBC). J Clin Oncol. 2018;36(15_suppl):TPS602-TPS602. doi:10.1200/JCO.2018.36.15_suppl.TPS602

64. Schmid P, Adams S, Rugo HS, et al. Atezolizumab and nab-paclitaxel in advanced triple-negative breast cancer. $N$ Engl J Med. 2018;379(22):2108-2121. doi:10.1056/NEJMoa1809615
65. Cortés J, Guo Z, Karantza V, Aktan G. KEYNOTE-355: randomized, double-blind, phase III study of pembrolizumab (pembro) + chemotherapy (chemo) vs placebo (PBO) + chemo for previously untreated, locally recurrent, inoperable or metastatic triple-negative breast cancer (mTNBC). J Clin Oncol. 2018;36(5_suppl):TPS18TPS18. doi:10.1200/JCO.2018.36.5_suppl.TPS18

66. Loi S, Giobbe-Hurder A, Gombos A, et al. Abstract GS2-06: phase Ib/II study evaluating safety and efficacy of pembrolizumab and trastuzumab in patients with trastuzumab-resistant HER2-positive metastatic breast cancer: results from the PANACEA (IBCSG 45-13/BIG 4-13/KEYNOTE-014) study. Cancer Res. 2018;78(4Supplement):GS2-06. doi:10.1158/15387445.SABCS17-GS2-06

67. Eng C. POLE mutations in colorectal cancer: a new biomarker? Lancet Gastroenterol Hepatol. 2016;1(3):176-177. doi:10.1016/ S2468-1253(16)30030-9

68. McConechy MK, Talhouk A, Leung S, et al. Endometrial carcinomas with POLE exonuclease domain mutations have a favorable prognosis. Clin Cancer Res. 2016;22(12):2865-2873. doi:10.1158/ 1078-0432.CCR-15-2233

69. Fader AN, Diaz LA, Armstrong DK, et al. Preliminary results of a phase II study: PD-1 blockade in mismatch repairdeficient, recurrent or persistent endometrial cancer. Gynecol Oncol. 2016;141:206-207. doi:10.1016/j.ygyno.2016. 04.532

70. Matulonis UA, Shapira-Frommer R, Santin A, et al. Antitumor activity and safety of pembrolizumab in patients with advanced recurrent ovarian cancer: interim results from the phase 2 KEYNOTE-100 study. J Clin Oncol. 2018;36(15_suppl):5511. doi:10.1200/JCO.2018.36.15_suppl.5511

71. Konstantinopoulos PA, Waggoner SE, Vidal GA, et al. TOPACIO/ Keynote-162 (NCT02657889): A phase $1 / 2$ study of niraparib + pembrolizumab in patients (pts) with advanced triple-negative breast cancer or recurrent ovarian cancer (ROC)-results from ROC cohort. J Clin Oncol. 2018;36(15_suppl):106. doi:10.1200/ JCO.2018.36.15_suppl.106

72. Le DT, Uram JN, Wang H, et al. PD-1 blockade in tumors with mismatch-repair deficiency. $N$ Engl $J$ Med. 2015;372 (26):2509-2520. doi:10.1056/NEJMoa1500596

73. Le DT, Durham JN, Smith KN, et al. Mismatch repair deficiency predicts response of solid tumors to PD-1 blockade. Science. 2017;357(6349):409-413. doi:10.1126/science.aan6733

74. Le DT, Yoshino T, Jäger D, et al. KEYNOTE-164: phase II study of pembrolizumab (MK-3475) for patients with previously treated, microsatellite instability-high advanced colorectal carcinoma. $J$ Clin Oncol. 2016;34(4_suppl):TPS787-TPS787. doi:10.1200/ jco.2016.34.4_suppl.tps787

75. Diaz LA, Le DT, Yoshino T, et al. KEYNOTE-177: phase 3, open-label, randomized study of first-line pembrolizumab (Pembro) versus investigator-choice chemotherapy for mismatch repair-deficient (dMMR) or microsatellite instability-high (MSI-H) metastatic colorectal carcinoma (mCRC). J Clin Oncol. 2018;36 (4_suppl):TPS877-TPS877. doi:10.1200/JCO.2018.36.4_suppl.TP S877

76. Shah MA, Bennouna J, Shen L, et al. Pembrolizumab for previously treated metastatic adenocarcinoma or squamous cell carcinoma of the esophagus: phase 2 KEYNOTE-180 study. $J$ Clin Oncol. 2016;34(15_suppl):TPS4139-TPS4139. doi:10.1200/ JCO.2016.34.15_suppl.TPS4139

77. Doi T, Bennouna J, Shen L, et al. KEYNOTE-181: phase 3, open-label study of second-line pembrolizumab vs singleagent chemotherapy in patients with advanced/metastatic esophageal adenocarcinoma. J Clin Oncol. 2016;34(15_suppl): TPS4140-TPS4140. doi:10.1200/JCO.2016.34.15_suppl.TPS 4140 
78. Ott PA, Piha-Paul SA, Munster P, et al. Safety and antitumor activity of the anti-PD-1 antibody pembrolizumab in patients with recurrent carcinoma of the anal canal. Ann Oncol. 2017;28 (5):1036-1041. doi:10.1093/annonc/mdx029

79. Fontugne J, Augustin J, Pujals A, et al. PD-L1 expression in perihilar and intrahepatic cholangiocarcinoma. Oncotarget. 2017;8:15. doi:10.18632/oncotarget.15602

80. Bang YJ, Doi T, Braud FD, et al. 525 Safety and efficacy of pembrolizumab (MK-3475) in patients (pts) with advanced biliary tract cancer: interim results of KEYNOTE-028. Eur J Cancer. 2015;51:S112. 10.1016/S0959-8049(16)30326-4.

81. Bauml J, Seiwert TY, Pfister DG, et al. Pembrolizumab for platinum- and cetuximab-refractory head and neck cancer: results from a single-arm, phase II study. J Clin Oncol. 2017;35 (14):1542-1549. doi:10.1200/JCO.2016.70.1524

82. Uppaluri R, Zolkind P, Lin T, et al. Neoadjuvant pembrolizumab in surgically resectable, locally advanced HPV negative head and neck squamous cell carcinoma (HNSCC). J Clin Oncol. 2017;35 (15_suppl):6012. doi:10.1200/JCO.2017.35.15_suppl.6012

83. Machiels J-PH, Licitra L, Rischin D, et al. KEYNOTE-412: pembrolizumab (pembro) in combination with chemoradiation versus chemoradiation alone in locally advanced head and neck squamous cell carcinoma (LA-HNSCC). J Clin Oncol. 2017;35(15_suppl): TPS6090-TPS6090. doi:10.1200/JCO.2017.35.15_suppl.TPS6090

84. Ghatalia P, Zibelman M, Geynisman DM, Plimack E. Approved checkpoint inhibitors in bladder cancer: which drug should be used when? Ther Adv Med Oncol. 2018. doi:10.1177/175883 5918788310

85. Kim S, Koh J, Kwon D, et al. Comparative analysis of PD-L1 expression between primary and metastatic pulmonary adenocarcinomas. Eur J Cancer. 2017;75:141-149. doi:10.1016/j.ejca.2017.01.004

86. Tretiakova M, Fulton R, Kocherginsky M, et al. Concordance study of PD-L1 expression in primary and metastatic bladder carcinomas: comparison of four commonly used antibodies and RNA expression. Mod Pathol. 2017;31:623. doi:10.1038/modpathol.2017.188

87. Robert C, Ribas A, Hamid O, et al. Durable complete response after discontinuation of pembrolizumab in patients with metastatic melanoma. J Clin Oncol. 2018;36(17):1668-1674. doi:10.1200/ JCO.2017.75.6270

88. Australian Pancreatic Cancer Genome Initiative, ICGC Breast Cancer Consortium, ICGC MMML-Seq Consortium, et al. Signatures of mutational processes in human cancer. Nature. 2013;500(7463):415-421. doi:10.1038/nature12477

89. Rizvi NA, Hellmann MD, Snyder A, et al. Mutational landscape determines sensitivity to PD-1 blockade in non-small cell lung cancer. Science. 2015;348(6230):124-128. doi:10.1126/science.aaa1348

90. Cristescu R, Mogg R, Ayers M, et al. Mutational load (ML) and T-cell-inflamed microenvironment as predictors of response to pembrolizumab. J Clin Oncol. 2017;35(7_suppl):1. doi:10.1200/ JCO.2017.35.7_suppl.1

91. Goodman AM, Kato S, Bazhenova L, et al. Tumor mutational burden as an independent predictor of response to immunotherapy in diverse cancers. Mol Cancer Ther. 2017;16(11):2598-2608. doi:10.1158/1535-7163.MCT-17-0386

92. Chalmers ZR, Connelly CF, Fabrizio D, et al. Analysis of 100,000 human cancer genomes reveals the landscape of tumor mutational burden. Genome Med. 2017;9:1. doi:10.1186/s13073017-0424-2

93. Lau E. Mismatch repair deficiency predicts benefit of anti-PD-1 therapy. Lancet Oncol. 2015;16(7):e319. doi:10.1016/S14702045(15)00031-5
94. Tazdait M, Mezquita L, Lahmar J, et al. Patterns of responses in metastatic NSCLC during PD-1 or PDL-1 inhibitor therapy: comparison of RECIST 1.1, irRECIST and iRECIST criteria. Eur $J$ Cancer. 2018;88:38-47. doi:10.1016/j.ejca.2017.10.017

95. Sachpekidis C, Hassel JC, Dimitrakopoulou-Strauss A. 18F-FDG PET/CT reveals disease remission in a patient with ipilimumab-refractory advanced melanoma treated with pembrolizumab. Clin Nucl Med. 2016;41(2):156-158. doi:10.1097/RLU.0000000000001039

96. Dercle L, Seban R-D, Lazarovici J, et al. 18F-FDG PET and CT scans detect new imaging patterns of response and progression in patients with hodgkin lymphoma treated by anti-programmed death 1 immune checkpoint inhibitor. J Nucl Med. 2018;59(1):15-24. doi:10.2967/ jnumed.117.193011

97. Dimitrakopoulou-Strauss A. Monitoring of patients with metastatic melanoma treated with immune checkpoint inhibitors using PETCT. Cancer Immunol Immunother. 2018. doi:10.1007/s00262-0182229-6

98. Muro K, Chung HC, Shankaran V, et al. Pembrolizumab for patients with PD-L1-positive advanced gastric cancer (KEYNOTE-012): a multicentre, open-label, phase 1b trial. Lancet Oncol. 2016;17 (6):717-726. doi:10.1016/S1470-2045(16)00175-3

99. Ayers M, Lunceford J, Nebozhyn M, et al. IFN- $\gamma$-related mRNA profile predicts clinical response to PD-1 blockade. J Clin Invest 2017;127(8):2930-2940. doi:10.1172/JCI91190

100. Herbst RS, Soria J-C, Kowanetz M, et al. Predictive correlates of response to the anti-PD-L1 antibody MPDL3280A in cancer patients. Nature. 2014;515(7528):563-567. doi:10.1038/nature 14011

101. Ribas A, Dummer R, Puzanov I, et al. Oncolytic virotherapy promotes intratumoral $\mathrm{T}$ cell infiltration and improves anti-PD-1 immunotherapy. Cell. 2017;170(6):1109-1119.e10. doi:10.1016/j. cell.2017.08.027

102. Conry RM, Westbrook B, McKee S, Norwood TG. Talimogene laherparepvec: first in class oncolytic virotherapy. Hum Vaccines Immunother. 2018;14(4):839-846. doi:10.1080/21645515.2017. 1412896

103. Milhem M, Gonzales R, Medina T, et al. Abstract CT144: intratumoral toll-like receptor 9 (TLR9) agonist, CMP-001, in combination with pembrolizumab can reverse resistance to PD-1 inhibition in a phase Ib trial in subjects with advanced melanoma. Cancer Res. 2018;78(13Supplement):CT144. doi:10.1158/1538-7445. AM2018-CT144

104. Brochez L, Chevolet I, Kruse V. The rationale of indoleamine 2,3-dioxygenase inhibition for cancer therapy. Eur $J$ Cancer. 2017;76:167-182. doi:10.1016/j.ejca.2017.01.011

105. Powles T, Bellmunt J, Petrylak DP, et al. Pembrolizumab (pembro) plus epacadostat or placebo for locally advanced or metastatic urothelial carcinoma (UC) after failure of first-line platinum-containing chemotherapy: KEYNOTE-698/ECHO-303. $J$ Clin Oncol. 2018;36(15_suppl):TPS4586-TPS4586. doi:10.1200/ JCO.2018.36.15_suppl.TPS4586

106. Balar AV, Plimack ER, Grivas P, et al. Phase 3, randomized, double-blind trial of pembrolizumab plus epacadostat or placebo for cisplatin-ineligible urothelial carcinoma (UC): KEYNOTE-672/ ECHO-307. J Clin Oncol. 2018;36(15_suppl):TPS4587-TPS4587. doi:10.1200/JCO.2018.36.15_suppl.TPS4587

107. Hamid O, Bauer TM, Spira AI, et al. Epacadostat plus pembrolizumab in patients with SCCHN: preliminary phase I/II results from ECHO-202/KEYNOTE-037. J Clin Oncol. 2017;35(15_suppl):6010. doi:10.1200/JCO.2017.35.15_suppl.6010 
108. Cohen EEW, Rischin D, Pfister DG, et al. A phase 3, randomized, open-label study of epacadostat plus pembrolizumab, pembrolizumab monotherapy, and the EXTREME regimen as first-line treatment for recurrent/metastatic head and neck squamous cell carcinoma (R/M SCCHN): ECHO-304/KEYNOTE-669. J Clin Oncol. 2018;36(15_suppl):TPS6090-TPS6090. doi:10.1200/ JCO.2018.36.15_suppl.TPS6090

109. Long GV, Dummer R, Hamid O, et al. Epacadostat (E) plus pembrolizumab $(\mathrm{P})$ versus pembrolizumab alone in patients (pts) with unresectable or metastatic melanoma: results of the phase 3 ECHO-301/KEYNOTE-252 study. $J$ Clin Oncol. 2018;36 (15_suppl):108. doi:10.1200/JCO.2018.36.15_suppl.108

110. Long GV, Atkinson V, Cebon JS, et al. Standard-dose pembrolizu$\mathrm{mab}$ in combination with reduced-dose ipilimumab for patients with advanced melanoma (KEYNOTE-029): an open-label, phase 1 b trial. Lancet Oncol. 2017;18(9):1202-1210. doi:10.1016/S14702045(17)30428-X
111. Ikeda M, Sung MW, Kudo M, et al. A phase $1 \mathrm{~b}$ trial of lenvatinib (LEN) plus pembrolizumab (PEM) in patients (pts) with unresectable hepatocellular carcinoma (uHCC). J Clin Oncol. 2018;36(15_suppl):4076. doi:10.1200/JCO.2018.36.15 suppl. 4076

112. Taylor MH, Rasco DW, Brose MS, et al. A phase $1 \mathrm{~b} / 2$ trial of lenvatinib plus pembrolizumab in patients with squamous cell carcinoma of the head and neck. $J$ Clin Oncol. 2018;36(15_suppl):6016. doi:10.1200/JCO.2018.36.15_suppl. 6016

113. Rodriguez $\mathrm{CP}, \mathrm{Wu} \mathrm{V}$, Voutsinas JM, et al. Phase I/II trial of pembrolizumab(P) and vorinostat $(\mathrm{V})$ in recurrent metastatic head and neck squamous cell carcinomas $(\mathrm{HN})$ and salivary gland cancer (SGC). J Clin Oncol. 2018;36(15_suppl):6025. doi:10.1200/ JCO.2018.36.15_suppl.6025

\section{Publish your work in this journal}

Cancer Management and Research is an international, peer-reviewed open access journal focusing on cancer research and the optimal use of preventative and integrated treatment interventions to achieve improved outcomes, enhanced survival and quality of life for the cancer patient.
The manuscript management system is completely online and includes a very quick and fair peer-review system, which is all easy to use. Visit http://www.dovepress.com/testimonials.php to read real quotes from published authors. 